\title{
DESCRIPCIÓN SOCIOAMBIENTAL DEL SUELO EN EL VALLE GEOGRÁFICO DEL RÍO CAUCA. EL CASO DE LA AGROINDUSTRIA AZUCARERA.
}

\author{
BRIGETTE TARYN CORTES ORTIZ ${ }^{1}$
}

Manizales, 2010-04-19 (Rev. 2010-05-12)

\section{RESUMEN}

El artículo tiene como propósito describir la forma en que el suelo en el Valle geográfico del río Cauca ha sido apropiado social y ambientalmente por el modelo agroindustrial del azúcar desde los inicios del siglo XX hasta la actualidad. En este sentido, el escrito hace un recorrido desde la economía hacendataria, que entró en crisis en el siglo XIX en lo que hoy es el Valle del Cauca hasta un modelo agroindustrial cada vez más complejo como lo es el cluster del azúcar.

El eje central del documento tiene que ver con la descripción histórico-espacial del desarrollo agroindustrial azucarero, el cual ha ejercido una fuerte presión en los recursos naturales principalmente lo que tiene que ver con el suelo, lo que permite evidenciar los diferentes impactos socioambientales que están definiendo la utilización y apropiación de este recurso en el Valle geográfico del río Cauca.

La metodología utilizada fue tomada de una propuesta de evaluación ambiental denominada "Presión/Estado/Respuesta", la cual constituyó el centro de registro y organización de la información recolectada del que surgieron diferentes indicadores cualitativos y cuantitativos, los cuales se integraron al trabajo documental realizado a partir diferentes documentos institucionales producidos por ASOCAÑA y CENICAÑA. Al mismo tiempo, lo que se busca es colocar este tema en el debate de las ciencias sociales, discutiendo sobre las nociones de desarrollo y ambiente, de acuerdo con las contribuciones de la producción intelectual de la Comisión Económica para América Latina (Citado en Sunkel, Cardoso \& Faletto 1981), Donald Worster (Citado en Castro,1995) Anthony Giddens (2000), y Ulrich Beck (1997, 1998a, 1998b).

\section{PALABRAS CLAVE:}

Sociología ambiental, descripción socioambiental del suelo, agroindustria azucarera, Valle del Cauca, aspectos ambientales.

\section{SOCIO-ENVIRONMENTAL DESCRIPTION OF THE GROUND IN THE VALLE DEL CAUCA. THE CASE OF THE SUGAR AGRIBUSINESS.}

\section{ABSTRACT}

The paper aims to describe how the ground in the Valle del Cauca has been socially and environmentally appropriate for the sugar agribusiness model from the early twentieth century to the present. In this sense, the research makes a journey from farm economy entered into crisis in the nineteenth century in what is 
now the Valle del Cauca to an agribusiness model increasingly complex as the cluster of sugar.

The core of the document deals with the historical-spatial description of the sugar agribusiness development, which has exerted strong pressure on natural resources which mainly has to do with the ground, which makes evident the different socio-environmental impacts are defining the use and ownership of this resource in the Valle del Cauca.

The methodology used was taken from a draft environmental assessment called "Pressure/Condition/Response", which was the center for registration and organization of the information collected from which emerged different qualitative and quantitative indicators, which joined the documentary work different from institutional documents produced by ASOCAÑA and CENICAÑA. At the same time, the aim is to place this issue in the discussion of the social sciences, discussing the notions of development and environment, according to the contributions of the intellectual output of the Economic Commission for Latin America (Called by Sunkel, 1981), Donald Worster (Called by Castro, 1995) Anthony Giddens (2000) and Ulrich Beck (1997, 1998a, 1998b).

KEY WORDS: environment sociology, description environment of the ground, agribusiness sugar, Valle del Cauca; aspects environment.

\section{APROXIMACIONES TEÓRICAS AL DEBATE AMBIENTAL. ¿ESTÁ PREPARADA LA SOCIOLOGÍA?}

Las formas de apropiación de la naturaleza por parte del ser humano han enfrentado el deterioro cada vez más frecuente de los recursos naturales, sobrepasando su función de reposición, depósito y soporte.

La gran promesa de un progreso ilimitado (la promesa de dominar la naturaleza, de abundancia material, de la mayor felicidad para el mayor número de personas y la libertad personal sin amenazas) ha sostenido la esperanza y la fe de la gente desde el inicio de la época industrial. (Fromm, 1991, p.21)

Estamos hoy enfrentados a un nuevo cambio de mentalidad, la fe en el progreso valuarte de la modernidad se está haciendo trisas gracias a las causas y consecuencias de la crisis ambiental que nos avisa la llegada a una de las paradojas más cruciales que afronta la humanidad, lo que Giddens (2000) llamó riesgos manufacturados los cuales intentan la aproximación a un campo de estudio nuevo que involucra lo social y natural al mismo tiempo, y en donde la indagación sobre el pasado y la reflexión que nos vislumbra el futuro muestran nuevos caminos para el abordaje del tema ambiental.

El panorama teórico que se presenta a continuación, inicia apoyado por los trabajos de investigación de la CEPAL y Donald Worster, que estudian el tema del desarrollo y su relación con el ambiente, a partir de lo que Castro (1995) denominó la naturaleza como construcción social.

La concepción acerca de que la naturaleza está ahí siempre va perdiendo su carácter, dado esto significa que sociedad y naturaleza se fusionan como dirían Beck et al. (1997) en una naturaleza social, lo natural está hoy inevitablemente unido a lo social. 
La concepción de una sociedad con un estado máximo de desarrollo empieza a tambalear, y el hecho se refleja en la peculiar mezcla entre naturaleza y sociedad, "la naturaleza se encuentra sometida y agotada a fines del siglo XX, y de ese modo ha pasado de ser un fenómeno exterior a un fenómeno interior, ha pasado de ser un fenómeno dado a ser un fenómeno producido" (Beck, 1998a, p. 19).

En primer término, la producción intelectual de la CEPAL organismo que desde su fundación y con lo que se constituyó como la teoría de la dependencia, fueron centrales para el análisis del desarrollo latinoamericano y que desde la década de los ochenta en el siglo XX establecieron permanentemente una reflexión teórica sobre las consecuencias ambientales del desarrollo económico.

En ese sentido, el desarrollo fue entendido por la CEPAL (Cardoso \& Faletto,1981) como "en sí mismo, un proceso social" de relaciones entre grupos, fuerzas y clases a través del cual algunos intentan imponer al conjunto del tejido social una forma de mantenimiento o propensión al cambio que le es propia, con todas las repercusiones políticas, sociales, culturales, económicas y ambientales consiguientes en el equilibrio de los grupos, tanto en el escenario local como en el global. Es a partir de este concepto que se discutirá el desarrollo producto de la consolidación de la agroindustria azucarera como centro hegemónico tanto agrícola como industrial en el Valle geográfico del río Cauca, especialmente en las zonas planas del departamento, extendiéndose a casi todo el suelo vallecaucano.

Junto a este planteamiento se encuentra el de Donald Worster, como uno de los mejores teóricos que constituyen un punto de partida en los conceptos fundamentales para el tratamiento de la historia natural.

Sin embargo, para entender cómo se ha ido produciendo la problemática ambiental, es necesario realizar una mirada retrospectiva en la que tentativamente Donald Worster (citado en Castro, 1995) ha desarrollado la Ilamada historia ambiental, debido a que pone en relieve fenómenos, problemas y temas que no se concebían hasta hace unas décadas.

Las condiciones ambientales están amenazando a la sociedad, por esta razón se hace necesario reevaluar la teoría sociológica tal como nos ha llegado del siglo XIX,

Lo sorprendente en ello es lo siguiente: los daños al medio ambiente y la destrucción de la naturaleza causada por la industria, con sus diversos efectos sobre la salud y la convivencia de los seres humanos se caracterizan por una pérdida del pensamiento social. A esta pérdida se añade lo grotesco: esta ausencia no le llama la atención a nadie, ni siquiera a los sociólogos. (Beck, 1998a, p. 31)

Sin embargo, la teoría de la modernización reflexiva plantea o sugiere examinar este tema cuestionando justamente la modernidad a partir de un tipo sui generis de seguridad ontológica; que consiste en que la confianza básica ya no se coloca en las certezas ofrecidas, sino en el conocimiento generado por sistemas abstractos.

Para Giddens (citado en Beck et al., 1997, p. 216), el núcleo de la tesis sobre el problema ambiental es la reflexividad, que contrapone la actitud ingenua de la modernización industrial, su optimismo respecto a la controlabilidad 
preestablecida de cosas incontrolables y puede construirse a partir de esto toda una cadena de argumentos, el claro ejemplo del optimismo tecnológico.

Es aquí donde aparece en escena la idea del riesgo que, a pesar de ser ampliamente utilizada por Ulrich Beck, ha sido definida por Anthony Giddens (2000) de la siguiente manera: el "riesgo no es igual a amenaza o peligro. El riesgo se refiere a peligros que se analizan activamente en relación a posibilidades futuras" (p. 35). "Por estas razones, la idea de riesgo siempre ha estado relacionada con la modernidad; pero quiero defender que en el periodo actual este concepto asume una nueva y peculiar importancia" (p. 38).

En ese sentido se resalta que existen dos tipos de riesgos:

El riesgo externo es el riesgo que se experimenta como viniendo del exterior, de las sujeciones de la tradición o de la naturaleza. Quiero distinguir éste del riesgo manufacturado, con lo que aludo al riesgo creado por el impacto mismo de nuestro conocimiento creciente sobre el mundo. (Ibíd.)

Los riesgos que se generan en el nivel más avanzado del desarrollo de las fuerzas productivas causan daños sistemáticos y a menudo irreversibles, suelen permanecer invisibles, se basan en interpretaciones causales,

pueden ser transformados, ampliados o reducidos, dramatizados o minimizados, por lo que están abiertos en una medida especial a los procesos sociales de definición. Con ello, los medios y las posiciones de la definición del riesgo se convierten en posiciones sociopolíticas clave. (Beck, 1998a, p. 28)

De este modo las fórmulas de generación de impactos ambientales de las ciencias naturales poseen un significado social, cultural y político.

La discusión amenaza con cometer el error contrario al error que con razón ha reprochado al optimismo de progreso industrial que durante mucho tiempo ha predominado: el error de convertirse en una discusión natural sin el ser humano, sin la cuestión del significado social y cultural. (Ibíd., p. 30)

Con la destrucción industrial de las bases ambientales de la vida se pone en marcha una dinámica social y política de desarrollo históricamente sin precedentes y que hasta ahora no ha sido comprendida, la cual nos obliga a repensar la relación entre naturaleza y sociedad. Para Beck (1998a) somos testigos de una fractura dentro de la modernidad, la cual se desprende de los contornos de la sociedad industrial clásica y desprende una nueva figura: la "sociedad del riesgo".

El riesgo manufacturado se refiere a situaciones que tenemos muy poca experiencia histórica en afrontar. La mayoría de los riesgos medioambientales, como los vinculados al calentamiento global, entra en esta categoría. Están directamente influidos por la globalización galopante. (Giddens, 2000, p. 42)

\section{METODOLOGÍA}

\section{Universo de estudio}


El universo de estudio es la Agroindustria Azucarera en el Valle geográfico del río Cauca -todo el Valle y el norte del Cauca-, teniendo en cuenta que es el cultivo permanente con más de doscientos mil hectáreas cultivadas en la zona y es la localización geográfica de más del $95 \%$ de los ingenios azucareros en Colombia.

\section{Estrategias de investigación}

\section{Análisis documental}

En la investigación se realizó un análisis de discurso, a partir de las publicaciones que realizan ASOCAÑA -Asociación de Cultivadores de Caña- y CENICAÑA Centro de Investigación de la Caña- en: boletines, folletos y documentación institucional, cuando hacen referencia al tema ambiental. También se tuvo en cuenta la documentación oficial de la Corporación Autónoma del Valle del Cauca -CVC-., de la Corporación de Cuencas Hidrográficas -CORPOCUENCAS- y del Departamento Administrativo de Planeación del Valle del Cauca-DAPV-, etc.

La investigación documental asumió como marco de referencia dos aspectos: la consolidación de la Agroindustria Azucarera, y el tratamiento al tema ambiental teniendo en cuenta igualmente dos subperiodos; a partir de la década de los noventa en el punto que ingresa con mayor vigor la legislación ambiental, y en segundo lugar desde que se firma el convenio de producción limpia con el Ministerio del Medio Ambiente en 1997 y el cual a pesar de la discusión que motivó en la esfera pública se ha prorrogado hasta la fecha, pero sirve como punto de referencia porque de todas maneras significó un viraje en la forma como este sector afrontaba el tema ambiental. El análisis de discurso tuvo en cuenta la procedencia de los planteamientos discursivos, de acuerdo a sus intereses, objetivos institucionales e históricos y el periodo de la publicación, de ahí se obtuvieron los indicadores cualitativos.

\section{Análisis de datos agregados}

La forma como se establecen los indicadores cuantitativos parte del registro y organización de los datos agregados que se realizaron a partir de la metodología ambiental Presión-Estado-Respuesta. El modelo de organización P-E-R fue desarrollado por Tony Friend y David Rapport, ampliamente conocidos por el desarrollo de indicadores ambientales desde 1979 (Winograd, 1994), lo que en su mayoría fueron datos técnicos que permitieron ampliar la visión que se tenía sobre la relación entre ambiente y sociedad que se pretende escudriñar aquí.

\section{DESCRIPCIÓN SOCIOAMBIENTAL DEL SUELO VALLECAUCANO}

En las primeras décadas del siglo XIX se produjeron grandes cambios en la estructura social y económica del Valle geográfico del río Cauca, debido a dos factores: el económico, surgido por la crisis de la minería y la caída de la exportación de oro; y el político, que gracias a las repetitivas guerras civiles socavaron la economía hacendataria.

La salida a esta crisis en la región se logra en la primera mitad del siglo XX con la aparición de la agroindustria tradicional; proceso que empezó desde mediados del siglo XIX con la reagrupación de antiguas haciendas coloniales, la dedicación 
de las tierras de la hacienda para el cultivo de productos como: el tabaco, la quina, el añil, el café y el azúcar, productos que ya tenían un mercado externo y que se constituirían en el engranaje del modelo agroexportador colombiano, al igual que formas de trabajo que fueron un síntoma de la transición y formación del desarrollo capitalista del campo.

De acuerdo con lo anterior, un elemento de suma importancia para la determinación de las particularidades del cultivo de caña de azúcar en la región tiene que ver con la forma de apropiación social de la tierra en las haciendas cañeras que dejó de ser solamente de apropiación social, es decir, una relación de propiedad meramente constituida como se menciona, para mediatizarse con una apropiación real, que permite en términos marxistas una acumulación originaria de capital.

"A partir de 1860 los procesos de producción de la caña y de su transformación en azúcar o en panela pasan a estar marcados por el papel innovador del ingenio 'La Manuelita', propiedad del inmigrante ruso-norteamericano James Eder" (Rojas, 1983, p. 18). La adquisición de la hacienda La Manuelita por parte de Santiago Eder y su socio Pío Rengifo, inició el cultivo de caña a gran escala en el Valle geográfico del río Cauca.

La configuración y consolidación de la agroindustria se da en dos momentos definitivos para la región. Un primer momento con el ingenio La Manuelita en el año 1901 hasta la década de los cincuenta, y un segundo desde 1960 hasta los momentos actuales, justamente son estos periodos los que lograrán determinar y describir los principales aspectos sociales y ambientales del suelo vallecaucano.

En la primera década del siglo XX la modernización del ingenio La Manuelita, fue un hecho que permitió elevar la producción a una nueva escala gracias a la maquinaria centrifuga importada de Europa presente en la fábrica. La década del veinte trae consigo el nacimiento de dos ingenios más: Providencia y Río Paila, gracias al gran auge de algunas condiciones económicas externas e internas.

Estas condiciones tienen que ver con la acumulación de capital desarrollada por el modelo agroindustrial azucarero, acumulación previa producto del comercio exterior, la consolidación del mercado interno de azúcar centrifugado, la accesibilidad geográfica a la región, el proceso de difusión tecnológica, la consolidación de proletariado agrario y la burguesía vallecaucana que logró constituirse como una clase social y empresarial dando origen inclusive a otras industrias diferentes a los ingenios azucareros.

La segunda década del siglo XX también es crucial para establecer la velocidad de la difusión tecnológica, en 1927 inicia actividades la Granja Experimental de Palmira entidad encargada de la investigación biológica y agrícola en general, y en el año 1929 llega a Colombia la misión puertorriqueña dirigida por Charles Chardon, que realizó el estudio Reconocimiento Agropecuario del Valle del Cauca, cuyo objetivo era analizar todas las características productivas y económicas del Valle geográfico del río Cauca, todo con el propósito de señalar que el "futuro" económico de la región estaría dado por el despliegue definitivo del cultivo de caña de azúcar, dadas las características biofísicas excepcionales del ambiente.

La década de los treinta se caracterizó por el inicio de labores de cuatro nuevos ingenios: Mayagüez, Bengala, María Luisa y La Industria aumentando sustancialmente el nivel de producción a 49.621 toneladas, pero es tal la complejidad del proceso de producción de estos ingenios de transición ${ }^{2}$, que aún 
tratándose de pequeñas escalas de producción aseguran la reproducción del proceso y la expansión económica de las empresas.

Como lo menciona Rojas (1983, p. 142), el complejo agroindustrial azucarero hunde sus raíces en la década de los cincuenta como segundo momento, en donde ingresan cinco ingenios más: La Cabaña, Meléndez, Tumaco, La Carmelita y San Fernando, con la producción de 276.812 toneladas al año. Hacia el periodo 1952-1953 ya había 22 ingenios azucareros en el Valle geográfico del río Cauca, de los cuales tres poseían extensiones aproximadas a 4.000 plazas cada una, cuatro tenían entre 2.000 y 4.000 plazas, y los restantes menos de 2.000 plazas.

Los ingenios azucareros incorporaron grandes fincas y lograron controlar en propiedad una considerable extensión de tierras y adaptaron nuevos métodos de cultivo $^{3}$, desde los sistemas de siembra, adecuación de tierras y riego, experimentación de variedades, tratamiento de semillas y control de plagas y malezas. Es en 1953 donde Manuelita S.A., que no olvida su papel de jalonador de todo el proceso de conformación de la agroindustria azucarera, marca el inicio de la fase de desarrollo agroindustrial con el montaje de una fábrica de refinación de azúcar, la cual supera su capacidad productiva en 2.500 toneladas día, manteniéndose indiscutiblemente como el ingenio de punta.

La actividad exportadora incrementa el ritmo productivo de la tierra y "la provisión de la materia prima pasa a ser un elemento definitivo de todo el ensanche o ampliación de fábrica tendiente a incrementar la participación de cada empresa en la producción del sector azucarero" (Rojas, 1983, p. 162), desencadenando una fuerte competencia por la tierra aún no cultivada de caña.

Los ingenios azucareros se vieron obligados a competir económicamente entre sí, ofreciendo pagos por la renta del suelo más ventajosos que los ofrecidos en ese momento por empresarios individuales dedicados a otros cultivos temporales o de corta duración.

La presión social por la tierra, que ejercieron los ingenios azucareros, se ve fielmente reflejada en las entrevistas realizadas en el libro Empresarios y tecnología en la formación del sector azucarero 1860-1980 escrito por el sociólogo José María Rojas, en el siguiente fragmento:

A toda esa negramenta los cogió y los envolvió a toditos con esas fincas. Le compraba eso o sino les quitaba el agua pa' que no hubiera producto. Él cogía por ejemplo: Usted tenía una finca allí y cogía y trazaba el agua por aquí y se venía por allí. Entonces el del café, el del plátano, el del maíz, como en ese tiempo de verano, pues que el agua, pues no hay agua. Ya el tipo se aburría. Pues a venderle todo eso. (1983, p. 122)

El control de las tierras que no eran parte de los ingenios tomó diversas formas como el arrendamiento y las cuentas de participación, así las tierras recuperan su autonomía administrativa y pasan a ser empresas productoras de caña ${ }^{4}$, proveedoras de los ingenios, de tal manera que las nuevas áreas dedicadas al cultivo de caña poseían los mismos cánones tecnológicos de los ingenios.

A finales de los años sesenta entran dos nuevos ingenios: El Naranjo y el hijo grande de Manuelita S.A., INCAUCA, quienes ayudan a mantener la producción regional necesaria para el mercado interno y participar consecutivamente en el mercado internacional, de igual modo, las cuotas estadounidenses cada vez 
fueron incrementándose haciendo que la producción aumentara de forma significativa.

Hasta aquí la configuración de la agroindustria azucarera no ha sido producto de un desarrollo lineal que apunta a una forma superior, sino que considera "la expansión del sector como un proceso que comporta la presencia de unidades empresariales extremadamente desiguales" (Ibíd., p. 115), lo cual se manifiesta principalmente con el control de tierras, mano de obra y por supuesto tecnología.

La agroindustria azucarera es todo un hecho, logrando integrar los diversos sectores de la economía en torno a su funcionamiento, además sus procesos de consolidación determinaron el contexto social, económico, político y cultural de la región.

La década de los ochenta llega con fuerza al complejo agroindustrial azucarero, la competencia por mantenerse en el negocio aceleró la concentración económica,

"la pequeña propiedad, allí donde la había, como en algunos municipios del norte y del sur del actual departamento del Valle, no podía resistir el embate de la expansión azucarera, pues la característica de esta expansión es su no articulación con las formas de producción campesina" (Ibíd., p. 32). También enfrentaron una intensa reconversión industrial y tecnológica y una reorganización social de la producción.

Esta fase de competencia sin precedentes llegó al escenario de la agroindustria azucarera con la Organización Ardilla Lulle acompañado por estilos de dirección más modernos, "gracias a la estrategia familiar, los azucareros de la élite mantuvieron con éxito el control sobre sus industrias hasta épocas recientes en que empiezan a aparecer fisuras en este modelo" (Urrea \& Mejía, 2000, p.101).

La supervivencia de los ingenios azucareros dependía décadas atrás de la política gubernamental y de la innovación empresarial de la región, varios de los cuales eran complejos familiares de gran tradición, éstos entrarían en una situación de incertidumbre no sólo por los cambios en los cuadros gerenciales, sino que el plan de desarrollo con el que iniciaba la década de los noventa en Colombia era un modelo de apertura económica que lanzaría la producción de azúcar a la competencia económica total.

Finalizando los años ochenta, la agroindustria azucarera se alistaba para incorporar al proceso de apertura, un modelo económico que buscaba atribuir al mercado la responsabilidad en la asignación de recursos mediante la eliminación de las acciones dirigidas, la desregulación y la limitación del Estado al establecimiento de reglas generales de operación del mercado, lo cual en un momento se pensó pondría en jaque el negocio del azúcar pero no fue así y prueba de ello fue el cluster del azúcar.

El último periodo es el correspondiente a los últimos 25 años. La concentración de tierras de los ingenios afiliados a ASOCAÑA corresponde a 2.029 unidades productivas (CENICAÑA, 1998a p. 74); es decir, los productores de caña y las hectáreas utilizadas para la siembra de caña de azúcar son 190.265 para 1998, no muy diferente a las 200.218 hectáreas al 2005, lo que indica que en siete años hay un incremento del 4,9\% aproximadamente y para el 2009 las áreas cultivadas eran 208.254 ha, lo que se observa en la siguiente tabla:

Tabla 1. Área Sembrada y Cosechada de Caña de Azúcar. 1986-2009 


\begin{tabular}{|c|c|c|c|c|c|}
\hline Año & $\begin{array}{c}\text { Area neta } \\
\text { sembrada } \\
\text { (hectáreas) }\end{array}$ & $\begin{array}{c}\text { Área } \\
\text { cosechada } \\
\text { (hectáreas) }\end{array}$ & Año & $\begin{array}{c}\text { Area neta } \\
\text { sembrada } \\
\text { (hectáreas) }\end{array}$ & $\begin{array}{c}\text { Area } \\
\text { cosechada } \\
\text { (hectáreas) }\end{array}$ \\
\hline 1986 & 139,092 & 106,729 & 1998 & 196,276 & 173,700 \\
\hline 1987 & 137,358 & 108,303 & 1999 & 197,354 & 167,100 \\
\hline 1988 & 138,608 & 111,964 & 2000 & 186,473 & 183,200 \\
\hline 1989 & 145,343 & 119,011 & 2001 & 192,572 & 174,160 \\
\hline 1990 & 152,427 & 122,038 & 2002 & 205,456 & 160,622 \\
\hline 1991 & 160,291 & 124,044 & 2003 & 198,038 & 170,002 \\
\hline 1992 & 165,226 & 126,913 & 2004 & 197,013 & 172,237 \\
\hline 1993 & 178,534 & 124,708 & 2005 & 198,049 & 176,367 \\
\hline 1994 & 181,063 & 133,730 & 2006 & 203,184 & 179,608 \\
\hline 1995 & 181,893 & 163,694 & 2007 & 202,926 & 184,866 \\
\hline 1996 & 184,039 & 178,026 & 2008 & 205,664 & 157,495 \\
\hline 1997 & 192,793 & 170,152 & 2009 & 208,254 & 192,744 \\
\hline
\end{tabular}

Fuente: Aspectos Generales del Sector Azucarero 2009-2010. ASOCAÑA.

Los ingenios con mayores unidades productivas son: en primer lugar, INCAUCA S.A., con 453 unidades productivas, y lo siguen Providencia S.A. con 324 y Central Castilla S.A. con 209; las dos primeras hacen parte de la Organización Ardila Lulle.

"Esta situación condujo a que Manuelita dejara de ser el líder histórico del sector y que drásticamente pasara del primero al tercer lugar de producción" (Urrea \& Mejía, 2000, p. 65), sin embargo, Manuelita S.A. posee las unidades productivas más extensas de todos los ingenios azucareros, ya que sólo posee 170 unidades productivas que siembran 19.943 hectáreas de caña.

Lo paradójico ocurre porqué "mientras en 1960 el 75\% del área pertenecía a los ingenios (aproximadamente 46.200 ha), en 1997 éstos sólo tenían el 24\% del área (44.112 ha); es decir, el área de los ingenios no ha crecido" (CENICAÑA, 1999a, p. 32). El cultivo de caña se convirtió entonces en un negocio en donde el control de tierras es efectuado por las empresas proveedoras de caña principalmente.

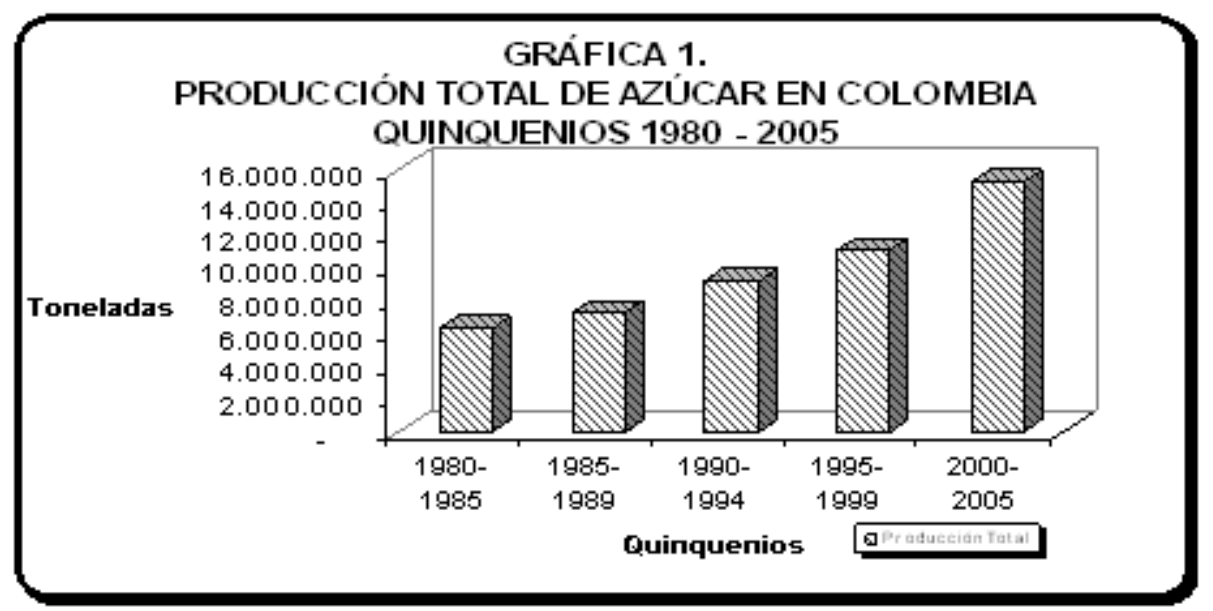


Fuente: Aspectos Generales del Sector Azucarero 2005-2006. ASOCAÑA.

La mayor producción de azúcar, de acuerdo a la Gráfica 1, responde básicamente a dos factores: primero, al continuo proceso de difusión tecnológica tanto en campo como en fábrica que mantienen los ingenios azucareros, que se sustenta en la investigación que por más de 20 años ha adelantado CENICAÑA. Segundo, un aumento importante de 13\% del área cosechada del cuarto (19951999) al quinto quinquenio (2000-2005) y de 2005 al 2009 la producción subió a 23 millones de toneladas.

La agroindustria azucarera es un sector cuya producción fue de más de dos millones de toneladas de azúcar en 1999, el incremento en la producción de azúcar ha sido sumamente significativo. Hace sólo treinta años la producción de azúcar era de 1'107.268 toneladas al año, para la década de los ochenta era de 1'247.488 toneladas (ASOCAÑA, 2000, p. 63), que representaba 140.220 toneladas adicionales. Para los noventa el tonelaje adicional subió a 368.963 toneladas y contra todos los pronósticos después de la apertura económica presenta 624.377 toneladas adicionales (Calero, 1996, p. 43).

\section{LOS IMPACTOS SOCIAMBIENTALES SOBRE EL SUELO VALLECAUCANO}

Los suelos en el Valle geográfico del río Cauca fueron agrupados en ocho clases agrológicas que se caracterizan por los atributos de la tierra de acuerdo al clima, pendiente, características físico-químicas, erosividad, drenaje y peligros de inundación. La distribución espacial de la zona plana según la clasificación agrológica de suelos presenta el $67,5 \%$ de las clases I, II y III que corresponden a los suelos más fértiles.

El porcentaje de participación de los suelos de la zona plana, que se encuentran en la Tabla 2, están representados así:

Tabla 2. Clasificación agrológica de suelos zona plana, Valle geográfico del río Cauca.

\begin{tabular}{|c|c|c|c|c|c|c|c|c|}
\hline \multirow[t]{2}{*}{$\begin{array}{l}\text { Clases de } \\
\text { suelos }\end{array}$} & \multicolumn{2}{|c|}{ Departamento } & \multicolumn{6}{|c|}{$\begin{array}{l}\text { Algunos municipios de Valle geográfico del } \\
\text { río Cauca }\end{array}$} \\
\hline & \multicolumn{2}{|l|}{ Valle $\%$} & \multicolumn{2}{|c|}{ Palmira \% } & \multicolumn{2}{|c|}{ Candelaria \% } & \multicolumn{2}{|c|}{ Cerrito \% } \\
\hline $\mathrm{T}$ & 34.796 & 11,0 & 5.928 & 14,9 & 4.380 & 14,1 & 1.795 & 7,3 \\
\hline II & 59.957 & 18,9 & 8.516 & 21,4 & 14.822 & 47,9 & 4.582 & 18,8 \\
\hline III & 83.901 & 26,5 & 7.210 & 18,1 & 9.072 & 29,3 & 7.635 & 31,3 \\
\hline IIIS & 207 & 0,0 & & & & & & \\
\hline $\mathrm{IIIH}$ & 1.214 & 0,3 & & & & & & \\
\hline IIIHS & 3.832 & 1,2 & & & & & 792 & 3,2 \\
\hline IV & 24.803 & 7,8 & 3.490 & 8,7 & & & 3.915 & 16,0 \\
\hline IVS & 9.544 & 3,0 & 308 & 0,7 & & & 2.205 & 9,0 \\
\hline IVSH & 35.107 & 11,0 & 270 & 0,6 & 200 & 0,6 & 252 & 1,0 \\
\hline V & 31.392 & 9,9 & 10.618 & 26,7 & 1.407 & 4,5 & 2.777 & 11,4 \\
\hline VSH & 5.932 & 1,8 & 1.982 & 4,9 & 890 & 2,8 & & \\
\hline $\mathrm{VH}$ & 6.093 & 1,9 & 92 & 0,2 & & & 100 & 0,4 \\
\hline Urbano & 18.412 & 5,8 & 1.336 & 3,3 & 145 & 0,4 & 265 & 1,0 \\
\hline Ciénaga & 1.133 & 0,3 & & & & & & \\
\hline Total & 316.323 & 100 & 39.750 & 100 & 30.916 & 100 & 24.318 & 100 \\
\hline
\end{tabular}


Fuente: SAG. Valle del Cauca. Tierras y Gentes. (1984, p. 15).

Tabla 3. Escalas de suelos del Valle del Cauca.

\begin{tabular}{|l|l|}
\hline $\begin{array}{l}\text { Clases de } \\
\text { suelos }\end{array}$ & Características \\
\hline I & $\begin{array}{l}\text { Son suelos de calidad óptima y pueden cultivarse } \\
\text { sin mayores dificultades, se trata de terrenos } \\
\text { planos no inundables ni fácilmente erosionables. }\end{array}$ \\
\hline $\begin{array}{l}\text { Suelos clase } \\
\text { II y III }\end{array}$ & $\begin{array}{l}\text { Al igual que los clase I son llanos, con leve } \\
\text { propensión a la erosión y no inundables. }\end{array}$ \\
\hline $\begin{array}{l}\text { Suelos clase } \\
\text { IV }\end{array}$ & $\begin{array}{l}\text { Utilizados para cultivos temporales o limitados, } \\
\text { pueden ser tanto más escarpados que los clase } \\
\text { III, están más desgastados y pueden ser } \\
\text { susceptibles a la erosión y tener menos fertilidad. }\end{array}$ \\
\hline $\begin{array}{l}\text { Suelos clase } \\
\text { V }\end{array}$ & $\begin{array}{l}\text { No son apropiados para los cultivos pero sí lo son } \\
\text { para las praderas y bosques. Estos suelos son } \\
\text { demasiados húmedos o pedregados como para } \\
\text { ser cultivados, pero sí pueden ofrecer forraje o } \\
\text { productos forestales. }\end{array}$ \\
\hline $\begin{array}{l}\text { Suelos clase } \\
\text { VI }\end{array}$ & $\begin{array}{l}\text { Son tierras apropiadas para la vegetación } \\
\text { permanente y su uso debe estar limitado a } \\
\text { pastoreo o a explotaciones forestales con } \\
\text { restricciones moderadas. Por tener un declive } \\
\text { marcado, estos suelos están expuestos a erosión, } \\
\text { Iluvias y vientos. }\end{array}$ \\
\hline $\begin{array}{l}\text { Su son apropiados para cultivos, se trata de } \\
\text { VII }\end{array}$ & $\begin{array}{l}\text { suelos escarpados, fácilmente erosionables, y } \\
\text { aunque pueden ser recomendados mejor para } \\
\text { bosques. }\end{array}$ \\
\hline $\begin{array}{l}\text { Suelos clase } \\
\text { VIII }\end{array}$ & $\begin{array}{l}\text { Sus tierras son francamente inapropiadas para } \\
\text { áridas o de pantanos de imposible desecación. } \\
\text { faunísticas silvestres. }\end{array}$ \\
\end{tabular}

Fuente: SAG. Valle del Cauca. Tierras y Gentes. (1984, p. 15).

De acuerdo a la Tabla 2, los suelos clase I se encuentran ubicados geográficamente en la zona plana, son tierras que poseen muy pocas limitaciones a corto plazo que restrinjan el uso agrícola, son adecuados para un margen amplio de plantas y cultivos, los cuales corresponden al $11 \%$ del territorio en zona plana.

El 19,9\% corresponden a la clase II, son tierras que tienen algunas limitaciones para los cultivos intensivos tecnificados o monocultivos, requieren prácticas cuidadosas de manejo y conservación, con leve propensión a la erosión y poco inundables.

Los suelos clase III son tierras que tienen algunas limitaciones para los cultivos intensivos tecnificados o monocultivos, requieren prácticas y obras especiales de 
conservación algo costosas de aplicar. El 28,1\% del territorio se encuentra en esta clasificación de suelos.

Los restantes $21,9 \%$ son suelos clase IV, los cuales son tierras con severas limitaciones que restringen la elección de plantas, requieren cuidadosas prácticas de manejo y conservación costosas de aplicar y mantener. Los suelos clase $\mathrm{V}$ son tierras con restricciones muy severas para los cultivos intensivos, las limitaciones son tales que el costo de corrección es muy alto y casi imposible de aplicar.

Los otros suelos presentes en el Valle geográfico del río Cauca, que corresponden al $14 \%$, tienen limitantes permanentes que en general son pendientes muy abruptas y suelos muy superficiales, aptos únicamente para la vegetación permanente de protección de vida silvestre o recreación.

En la Tabla 1, podemos encontrar que en la clasificación agrológica del Valle geográfico del río Cauca los suelos más fértiles son los clase I, II y III, con gran representatividad dentro de los municipios de Palmira, Candelaria y Cerrito, los cuales tienen las principales tres áreas más sembradas de Caña de Azúcar del departamento -32.175 ha, 20.529 ha y 13.698 ha, respectivamente-, poseen entre 2.000 a 4.000 ha de suelos clase I; los suelos clase II se encuentran mayoritariamente en Palmira y Candelaria entre 6.000 y 12.000 ha y los suelos clase III se encuentran en los municipios de Candelaria, Palmira y Cerrito con 8.000 ha aproximadamente en cada municipio.

En cuanto a los patrones de uso, la agricultura vallecaucana experimenta una notable industrialización producto de la tecnificación, que encuentra en las plantaciones de caña las más grandes e importantes del país; los cultivos permanentes encontrados en el departamento son: Caña de Azúcar, 185.806 ha; Café, 90.759 ha; Plátano, 17.132 ha; Caña panelera, 5.835 ha, y Cacao, 413 ha, entre los más importantes (DAPV, 2004).

La presión del cultivo de caña de azúcar sobre los suelos denominados por el mismo discurso gremial azucarero como "tierras excepcionalmente fértiles del Valle del Cauca", es enorme principalmente en los municipios de Palmira y Candelaria, los cuales tienen tres características básicas: mayor área sembrada de caña de azúcar, más suelos fértiles con respecto a los otros municipios del departamento, y son los que más conflicto socioambiental poseen.

No obstante, es sólo un $11 \%$ el que puede considerarse como tierras "excepcionalmente fértiles", las cuales no tendrán problemas ambientales (a menos a corto y mediano plazo), soportando el monocultivo de la caña de azúcar el resto del suelo vallecaucano el cual se vuelve propenso a la degradación propia del monocultivo.

Este fenómeno de la artificialización de los cultivos viene acompañada del problema de la especialización de los suelos que surge como consecuencia del objetivo de maximizar la productividad de un recurso (y muy relacionado también con la idea de ventaja comparativa como principio de la política de desarrollo). La especialización puede derivar en mayor productividad, pero también genera condiciones de mayor fragilidad, algo bien conocido en la agricultura. (Sunkel, 1981, p. 33)

El recurso natural del suelo es el referente más claro sobre cómo los empresarios cañicultores convirtieron a éste en un factor que determinaba la organización 
social dada la escasa capacidad de movilidad social, determinando aún más el monocultivo. La adecuación de la tierra para la caña de azúcar y sus prácticas permanentes de mecanización y uso de implementos tecnificados de labranza, corte y cosecha, han degradado los suelos pulverizándolos superficialmente y compactándolos más internamente, generando impactos ambientales en el suelo como: la salinización, el drenaje, la pérdida de estructura, la compactación y la pérdida de potencial productivo por lixiviación, y otros problemas referidos a la contaminación por agroquímicos y residuos.

La desertificación es también un impacto de la agricultura tecnificada, es la provocada por las prácticas de riego. De acuerdo con lo anterior, se presentan dos situaciones: si el suelo no se acumula, logra depurar los excesos y regula un equilibrio finito con respecto a las características de los mismos; si el suelo se acumula y llega a un punto de saturación se producen fenómenos como la salinización, lixiviación y compactación.

Un ejemplo al respecto, es que el $43,2 \%$ del área en zona plana tiene problemas de salinización y drenaje, es decir, el $28,6 \%$ por salinidad y por drenaje el $22,7 \%$. El municipio con mayor afectación por salinidad en el Valle geográfico del río Cauca es Palmira con un $50,5 \%$ de su superficie, y en cuanto a los problemas de drenaje con un $42,7 \%$ el municipio de Guacarí. También hay que tener en cuenta la utilización de maquinaria agrícola que se desplaza por todo el área del cultivo, la cual es sumamente pesada y genera compactación del terreno.

Otra de las consecuencias de la quema de follaje es la que se produce en el suelo, implica un alto impacto que despoja a la tierra de nutrientes y humedad disminuyendo su fertilidad que en condiciones normales generan los residuos de las hojas de la caña al caer al suelo.

De igual manera, se presentan pérdidas de nutrientes al suelo cuando la ceniza cae a él y lo saliniza; mientras no existan los efectos benéficos que aportan la descomposición de los residuos de las hojas de caña de azúcar, la tierra seguirá careciendo de elementos nutrientes que le ayuden a conservar su fertilidad.

\section{LA RESPONSABILIDAD SOCIAL Y AMBIENTAL DE LA AGROINDUSTRIA AZUCARERA}

La responsabilidad permite ser competitivo en el tiempo y tener permanencia en la actividad. Preocuparse por el entorno es pues, de acuerdo con Ricardo Villaveces Pardo, la manera más idónea de garantizar unas condiciones para hacer bien una actividad que se desarrolla a la vez que se logra la solidaridad con el sector, porque la gente participa de él. (ASOCAÑA, 1998, p. 7)

Esta actividad de "solidaridad" es más bien como lo anotan Urrea y Mejía (2000) una práctica filantrópica, producto de la transfiguración de los sistemas paternalistas utilizados desde el comienzo de la actividad azucarera, y que se han enmarcado en una nueva función de intervención social asistencialista ayudando a las comunidades en diferentes programas y acciones como: programas de vivienda, salud, educación, actividades lúdicas, etc.

Sin embargo, los esfuerzos valiosos pueden ser insuficientes sino se consolida un desarrollo social, económico y cultural que integre a la población al conjunto de la vida social regional, con criterios de equidad social y con una oferta de 
bienes y servicios que contribuya a la creación y desarrollo de espacios públicos. (Urrea \& Mejía, 2000, p. 128-129)

Para la organización gremial azucarera la responsabilidad con el ambiente y la manera de hacer participe a las poblaciones radica en la inversión ambiental, que incluye: estudios, obras, operación y mantenimiento de equipos de producción, divulgación y capacitación, cifra que al 2005 -desde 1995 a 2005- rodea los \$ 270.373'000.000 (ASOCAÑA, 2005).

La inversión ambiental que realizan los ingenios azucareros a pesar de ser alta, necesaria e importante, no muestra una convicción real frente al pensar, concebir y planear en el largo plazo el futuro del ambiente y el desarrollo en la región. De esta forma, el sector empresarial se enfrenta a un doble reto ante el objetivo ambiental; por una parte, incorpora el componente ambiental en la estrategia de la empresa realizando las inversiones necesarias en investigación y desarrollo de tecnologías limpias, la modificación de procesos, las medidas correctivas, la gestión de residuos, etc.; y por otra, deja las bases sólidas para que los pobladores rurales mejoren su calidad de vida.

Es necesario que las empresas planifiquen toda una estrategia de desarrollo en la que se detecten los problemas pasados, presentes y futuros que la actividad acarrea al ambiente. A tal fin, es menester traspasar las fronteras sectoriales y disciplinarias, temporales y espaciales para abordar la realidad con su interdependencia sistémica, sus límites y su complejidad.

Lograr la armonía entre el ambiente y las actividades económicas dependerá en una pequeña parte del avance tecnológico respecto a la protección ambiental, pero fundamentalmente deberá producirse un cambio serio y profundo en toda la sociedad, sin excepciones en cuanto a las actitudes y los compromisos hacia el ambiente, es decir, es necesario construir una cultura ambiental.

La responsabilidad social y ambiental de las empresas dedicadas al cultivo de la caña de azúcar, deberá girar en torno a un nuevo valor social propio de un nuevo paradigma de racionalidad económica, condicionada por una racionalidad con arreglo a valores en vez de privilegiar la racionalidad los fines.

\section{DISCUSIÓN}

En la medida en que se incremente el área cultivada con plantas de la misma especie como el cultivo de caña de azúcar en el Valle geográfico del río Cauca, hay una intranquilidad latente en los pobladores rurales con un nuevo ingrediente: la degradación ambiental que amenaza y pone en peligro la calidad de vida, afectando cada vez más la calidad y cantidad de los suelos del característico ritmo con que la actividad agrícola busca la alta productividad a corto plazo.

Si antes fueron las haciendas hoy los ingenios continúan la expansión, pues las fumigaciones aéreas nos acaban, aunque dizque son para la caña. Eso acaba con todo lo que uno tiene sembrado, cuenta Santiago Rodríguez. Agrega que además del gusano "pasador", los pesticidas terminaron hace unos años con el cacao. (Hoyos, 1996, p.18) 
El ritmo de la industrialización de la caña de azúcar hace que el problema de la eficiencia sea un propósito cada vez más complejo, debido principalmente a la compleja incertidumbre que acompaña el uso de los recursos naturales en las regiones con más alta presión sobre ellos.

La agricultura intensiva expandida hoy en día no es indefinidamente sostenible. Utiliza grandes cantidades de fertilizantes e insecticidas químicos, destructivos, para el medio ambiente. No podemos volver a los modos más tradicionales de cultivar la tierra y esperar todavía alimentar a la población mundial. (Giddens, 2000, p. 46)

Los efectos colaterales y autoamenazas causados por el deterioro ambiental que ha permanecido y aún algunos permanecen invisibles, son producidos de forma sistemática y a menudo irreversibles, pero al convertirse en temas de debate público fueron minimizados, reducidos o disminuidos en el centro de los conflictos políticos de otro orden, el ambiental.

"Esto coincide con el nuevo paradigma de la sociedad del riesgo, que para su núcleo reposa en la solución de un problema similar y sin embargo, completamente diferente". (Beck, 1998a, p. 26) Estas evidencias se hacen palpables con la nueva figura de la sociedad del riesgo, donde las amenazas invisibles del impacto ambiental y el posterior deterioro de la calidad de vida de los pobladores rurales quedan visibles.

En consecuencia, la sociedad del riesgo dispone de nuevas fuentes de conflicto y consenso; es verdad que crecen las amenazas pero son transformadas políticamente en una política preventiva de la dominación del riesgo, pero quien los crea debe asumirlos con coraje y aventura.

En el Valle geográfico del río Cauca, los peligros invisibles que hasta ahora genera el desarrollo agroindustrial azucarero se volverán visibles cuando la fase de latencia de las amenazas del riesgo llegue a su fin, los daños y destrucciones a la naturaleza ya no se originan solamente fuera de la experiencia personal en la esfera física, química o biológica de las cadenas de efectos, sino que cada vez saltan con mayor claridad a la vista, al olfato y al oído.

El final de la latencia tiene dos caras: el riesgo mismo y su percepción pública; es conocido que en la región vallecaucana esta fase se encuentra en la primera etapa de la caracterización del riesgo, nunca queda claro si los riesgos se han intensificado o lo han hecho nuestra visión de ellos.

La amenaza ambiental para la población rural del Valle geográfico del río Cauca está enfocada en dos aspectos fundamentales: el desplazamiento o expulsión de la población campesina por la ampliación de la frontera agrícola y la concentración del cultivo de caña para los que puedan acceder a tecnología de punta; y en segunda instancia, la gran cantidad de pobladores rurales que dependen del cultivo pero que cada vez el mismo se está desprendiendo de ellos, como lo que sucede con los corteros de caña los cuales perderán sus trabajos cuando se consolide la mecanización del corte de caña.

\section{REFERENCIAS BIBLIOGRÁFICAS}


- Asociación de Cultivadores de Caña. (1997). Desarrollo del convenio de producción limpia del sector azucarero. Boletín ASOCAÑA, 20, 4-7. . (1998a). Diagnóstico ambiental. Impacto generado por las actividades de elaboración de azúcar en el recurso aire. Boletín ASOCAÑN, 23, 6-9.

- (1998b). La responsabilidad ambiental como parte del negocio. Boletín ASOCAÑA, 26, 4-5. - $\quad$ (2000). Aspectos generales del sector azucarero 2000-2001. Cali: ASOCAÑA. . (2005). Aspectos generales del sector azucarero 2005-2006. Cali: ASOCAÑA. - $\quad$ (2009). Aspectos generales del sector azucarero 2009-2010. Cali: ASOCAÑA.

- $\quad$ Beck, Ulrich. (1998a). La sociedad del riesgo. Hacia una nueva modernidad. Barcelona: respuestas a la globalización. Barcelona: Paidós. (1998b). ¿Qué es globalización? Falacias del globalismo, - Beck, Ulrich et al. (1997). Modernización reflexiva. Política, tradición y estética en el orden social moderno. Madrid: Alianza Editorial. - Calero, Alba Patricia. (1996). Impacto ambiental ocasionado por la quema de follaje de la caña de azúcar. Facultad de Humanidades, Trabajo de Grado, Programa de Licenciatura en Ciencias Sociales, Universidad del Valle, Cali - Cardoso, Fernando y Faletto, Enzo. (1981). Dependencia y desarrollo en América Latina. Ensayo de interpretación sociológica. Bogotá: Siglo XXI. - Castro, Guillermo. (1994). Los Trabajos de ajuste y combate: Naturaleza y sociedad en la historia de América Latina. Bogotá: Ediciones Casa de las Américas.

- (1995). Problemas y paisajes. Campus Virtual CLACSO, Argentina. - Centro de Investigación de la Caña de Azúcar. (1997). Informe anual 1997. Cali.

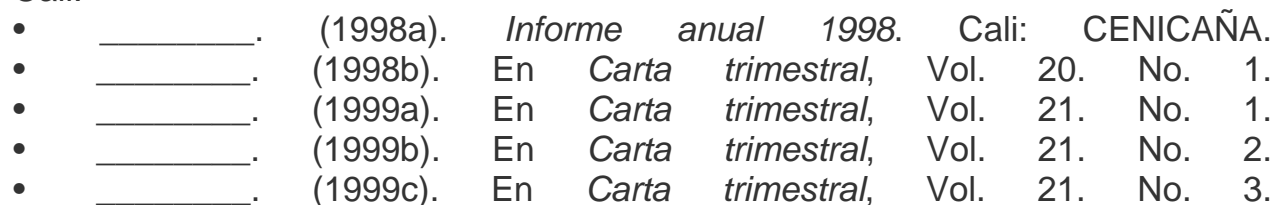
- Colmenares, Germán. (1983). Terratenientes, mineros y comerciantes. Bogotá: Universidad del Valle - Banco Popular. - Corporación Autónoma del Valle del Cauca. (1997a). Plan de gestión ambiental para el Valle del Cauca. Una visión de futuro. Cali: Corporación Autónoma del Valle del

Cauca. - (1997b). Cifras de tierra y vida. Cifras del medio ambiente en el Valle del Cauca 1995-1997. Cali: Corporación Autónoma del Valle del Cauca. - Cortes, Brigette Taryn. (2002). Implicaciones socioambientales del desarrollo agroindustrial cañero en el distrito No.2 del valle geográfico del río Cauca. Facultad de Ciencias Sociales y Económicas, Trabajo de Grado, Programa de Sociología, Universidad del Valle, Cali - Departamento Administrativo de Planeación Valle del Cauca. (2004). Anuario estadístico del Valle del Cauca. Cali: Departamento Administrativo de Planeación del Valle del Cauca. - Fromm, Erich. (1991). ¿Tener o ser? México: Fondo de Cultura Económica. - Giddens, Anthony. (2000). Un mundo desbocado. Los efectos de la globalización en nuestras vidas. Madrid: Ediciones Taurus. - Hoyos, Guido. (1996). El Valle de los campesinos de ciudad. En El País de Cali, Gaceta Dominical, Febrero 18 de 1996. - Rojas, José María. (1983). Empresarios y tecnología en la formación del sector azucarero en Colombia 1860-1980. Bogotá: Universidad del Valle - Banco Popular. 
- Sociedad de Agricultores y Ganaderos del Valle del Cauca. (1984). El Valle del Cauca. Tierras y gentes. Una visión de su actividad agropecuaria. Cali: Sociedad de Agricultores y Ganaderos del Valle del Cauca. - Sunkel, Osvaldo. (1981). La dimensión ambiental en los estilos de desarrollo de América Latina. Santiago de Chile: CEPAL - PNUMA. - Urrea, Fernando y Mejía, Carlos. (2000). Innovación y cultura de las organizaciones en el Valle del Cauca. En Urrea, Fernando et al., (Comps.), Innovación y cultura en tres regiones de Colombia. (pp. 83-218). Bogotá: Colciencias - c Corporación Calidad. - Winograd, Manuel. (1994). Environmental indicator for Latin America and the Caribbean: Toward land-use sustainability. Estados Unidos: World Resources Institute.

1. Socióloga, egresada de la Universidad del Valle. Actualmente tesista de la Maestría "Teoría y Metodología en la Ciencias Sociales" de la CLACSO y estudiante de la Maestría en Hábitat de la Universidad Nacional sede Manizales. Docente Ocasional, Departamento de Antropología y Sociología, Universidad de Caldas, Manizales, Colombia.

2. Es la tipificación de los ingenios que pasan de la producción de panes de azúcar al azúcar centrifugado, lo cual implicaba pasar de la hacienda azucarera tradicional a esta forma empresarial denominada "ingenio de transición".

3. La Escuela Superior de Agricultura Tropical, que luego se transformaría en la Facultad de Agronomía en Palmira, se dedica a labores de carácter docente, investigativo y técnico que beneficiaron la naciente agroindustria azucarera.

4. Algunas empresas productoras de caña fueron grandes extensiones de tierra que no alcanzaron a convertirse en ingenios azucareros debido a la falta de acumulación de capital. 